\title{
Selected papers presented at the Symposium on Nutrition and Metabolism of the Brazilian Society for Development of Research in Surgery (SOBRADPEC)
}

\author{
Paulo Roberto Leitão de Vasconcelos ${ }^{\mathrm{I}}$, Sérgio Botelho Guimarães ${ }^{\mathrm{II}}$ \\ ${ }^{\mathrm{I}} \mathrm{PhD}$, Associate Professor, Coordinator, Post-Graduation Program, Department of Surgery, Federal University of Ceará (UFC), Brazil \\ ${ }^{\text {II }} \mathrm{PhD}$ Associate Professor and Head, Surgical Research Laboratory (LABCEX), Department of Surgery, Federal University of Ceará (UFC)
}

The Brazilian Society for Development of Research in Surgery (SOBRADPEC) has been playing a most relevant role to the development and implementation of research at various research centers in Brazil. The papers contained in this Supplement were presented at a Symposium organized by the State of Ceará Regional of SOBRADPEC, Brazil, coordinated by Prof. Luiz Gonzaga Porto Pinheiro, President of the Regional.

All papers included in this Supplement represent original research presented as manuscripts or thesis to the Program of Post-Graduation Stricto Sensu in Surgery of the Federal University of Ceará (UFC). They address the use of nutraceutics, which are isolated or combined nutrients offered at pharmacological doses not found in a normal diet. This means nutrition modifying the biological response to stress promoting immune enhancement and metabolic protection. Of the seventeen papers here presented four are clinical investigations whereas the others are experimental.

Nutraceutics were used either as a preconditioning tool or as a treatment to change the metabolic/oxidative stress response to trauma and ischemia/reperfusion lesion. Besides nutraceutics, a new nitric oxide donor and anaesthetic drugs have been studied. The impact of acupuncture upon metabolism and oxidative stress has also been investigated. Three experimental models on thermal injury and remote pulmonary lesion were also included. 